\title{
Estudo da secção do ligamento carpal transverso comparando as técnicas endoscópica e convencional em cadáver humano ${ }^{1}$
}

\author{
José Estrela Neto ${ }^{2}$ \\ José Alberto Dias Leite ${ }^{3}$ \\ Marcelo José Cortez Bezerra ${ }^{4}$
}

\begin{abstract}
Estrela Neto J, Leite JAD, Bezerra MJC. Estudo da secção do ligamento carpal transverso comparando as técnicas endoscópica e convencional em cadáver humano. Acta Cir Bras [serial online] 2003 Mar-Abr;18(2). Disponível em URL: http://www.scielo.br/acb.
\end{abstract}

RESUMO - Objetivo: Verificar, em cadáver, a secção do ligamento carpal transverso (LCT) comparando as técnicas endoscópica tipo CHOW e convencional tipo mini-incisão. Métodos: Foram utilizados 18 cadáveres, não formolizados, perfazendo um total de 36 punhos, distribuídos em dois grupos. O grupo I compreendeu os 18 punhos esquerdos e utilizou a técnica endoscópica, enquanto o grupo II incluiu os 18 punhos direitos e empregou a técnica convencional. Resultados: A secção completa do LCT ocorreu em 15 casos (83,3 \%) no grupo I e em 17 casos $(94,4 \%)$ no grupo II. O tempo operatório no grupo I teve uma média de 27,2 minutos, enquanto no grupo II o tempo médio foi de 29,7 minutos. Verificou-se lesão do nervo mediano em dois casos $(11,1 \%)$ e somente no grupo I. Conclusão: Não há diferença significante no que se refere à secção do LCT e ao tempo operatório quando se comparam as técnicas endoscópica tipo Chow e convencional tipo miniincisão. A incidência de lesão do nervo mediano, porém, é significante $(\mathrm{p}<0,05)$ quando se utiliza a técnica endoscópica, o que pode estar relacionado à curva de aprendizagem.

DESCRITORES - Síndrome do túnel carpal. Técnica endoscópica versus convencional.

\section{Introdução}

O ligamento carpal transverso (LCT) também denominado retinaculum flexor é um espessamento transverso da fáscia profunda do antebraço, que ao se inserir, lateral e medialmente, nos ossos do carpo, constitui o teto do túnel carpal que contém os tendões flexores dos quirodáctilos e o nervo mediano, cuja compressão produz a síndrome do túnel carpal. Esta é a neuropatia periférica mais diagnosticada e tratada no mundo, incide em cerca de $1 \%$ na população geral e em $5 \%$ dos trabalhadores que requerem uso repetitivo das mãos ${ }^{1}$.
A compressão do nervo mediano pode ser causada por qualquer alteração que reduza de modo significante o tamanho do túnel carpal, como por exemplo, artrite reumatóide, cisto sinovial e lesão por esforço repetitivo. Os sintomas e sinais variam desde os mais precoces, como a dor noturna, ao mais tardio como a hipotrofia tenar. No insucesso do tratamento conservador, para aliviar os sintomas e sinais da síndrome do túnel do carpo, faz-se à secção do LCT, operação denominada descompressão do túnel do carpo, utilizando-se os procedimentos convencional ou endoscópico ${ }^{2,3}$. O procedimento cirúrgico convencional, também

1. Trabalho realizado no Programa de Pós-graduação Stricto Sensu do Departamento de Cirurgia da Faculdade de Medicina da Universidade Federal do Ceará (DC/FM/UFC).

2. Prof. Adjunto do DC/FM/UFC e Mestrando em Cirurgia.

3. Livre Docente e Professor do Programa de Pós-graduação do DC/FM/UFC.

4. Membro da Sociedade Brasileira de Ortopedia e Traumatologia e Mestrando em Cirurgia. 
denominado aberto, consiste em se empregar uma incisão longitudinal no punho que se inicia na prega proximal e adentra a região palmar, podendo ser longa ou curta. A incisão longa, chamada convencional, mede cerca de $4,5 \mathrm{~cm}^{4}$ podendo chegar a $9,0 \mathrm{~cm}^{5}$, enquanto a incisão curta ou pequena, denominada miniincisão, mede cerca de $2,5 \mathrm{~cm}^{4,6}$ a $1,5 \mathrm{~cm}^{7}$. Em profundidade, incisam-se todas as camadas da pele, depois, o LCT, quando o túnel do carpo é aberto e, portanto, descomprimindo, com visão direta. No procedimento endoscópico emprega-se a visão indireta através de instrumentos de artroscopia modernos ${ }^{8}$, que pode ser introduzido por uma incisão, método uniportal de Agee $^{9}$ ou duas incisões, método biportal de $\mathrm{Chow}^{10}$, medindo aproximadamente $1,0 \mathrm{~cm}$ localizadas no punho e na palma da mão.

Apesar de existirem vários trabalhos que defendem a secção endoscópica como técnica segura e sem complicações ${ }^{10}$, outros estudos mostram que a técnica convencional é mais eficiente ${ }^{11}$ ou, ainda, que ambas as técnicas convencional e endoscópica são eficientes ${ }^{12}$, porém todos são trabalhos clínicos e, como tais, não comprovam a real secção do ligamento carpal transverso. Por isso, decidiu-se analisar, a secção do ligamento carpal transverso comparando as técnicas convencional e endoscópica em cadáver humano.

\section{Métodos}

Foram utilizados 18 cadáveres humanos, não formolizados e não reclamados, perfazendo um total de 36 punhos, obtidos no Instituto Médico Legal do Estado do Ceará, obedecendo às normas que regulamentam a pesquisa em seres humanos, do Conselho Nacional de Saúde - Ministério da Saúde, Resolução no. 196 de 10.10.1996, e devidamente aprovado pelo Comitê de Ética e Pesquisa do Complexo Hospitalar da UFC - COMEPE (Anexo A). Eram de ambos os sexos, sendo 15 masculinos $(83,3 \%)$ e três femininos $(16,6 \%)$. A faixa etária aparente variou entre 20 e 45 anos, com idade média aparente de 27,5 anos. Com relação à raça, sete eram brancos $(38,8 \%)$, oito eram mestiços $(44,4 \%)$ e três pertenciam à raça negra $(16,6 \%)$.

Instrumental cirúrgico - Usaram-se os seguintes aparelhos na técnica convencional: paquímetro, canetas demográficas para pele, cabo de bisturi número três, lâmina de bisturi número 15 , tesoura de dissecção, tesoura reta, pinça de Adson com dente, porta agulha, afastador de Farabeuf e mini-afastador autostático; enquanto na técnica endoscópica foram utiliz ados estabilizadores de voltagem, gancho com lâmina cortante reversa, palpador, trocarte, cânula fenestrada, fonte de luz, óptica do artroscópio com lente angular de $30^{\circ}$ para liberação do túnel carpal (DYONICS $\left.{ }^{\circledR}, \mathrm{USA}\right)$, câmera, monitor de televisão de 14 polegadas (Figura 1).

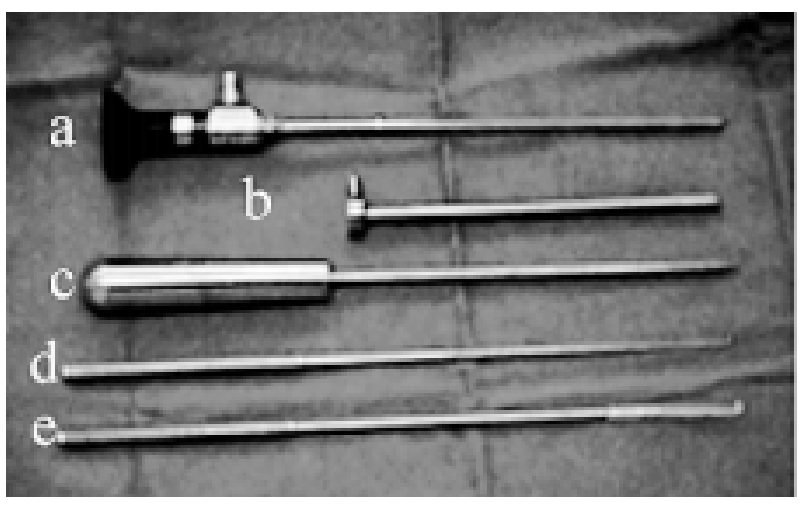

FIGURA 1 - Instrumental endoscópico:

a) óptica do artroscópio; b) cânula fenestrada; c) trocarte; d) palpador; e) gancho com lâmina cortante reversa

\section{Procedimentos}

Distribuição dos cadáveres: grupo I constituído por 18 (dezoito) punhos esquerdos operados pela técnica endoscópica tipo Chow, e grupo II, constituído por 18 dezoito punhos direitos operados pela técnica convencional tipo mini-incisão.

Critérios de inclusão: todos os cadáveres eram adultos e os punhos não apresentavam cicatriz, trauma ou feridas contusas.

Critério de avaliação: considerou-se secção completa quando não havia qualquer resquício do ligamento carpal transverso.

Técnica operatória: na convencional ${ }^{6}$ empregouse uma incisão longitudinal com cerca de $2,5 \mathrm{~cm}$ (Figuras 2 e 3), iniciando-se próximo à prega cutânea distal do punho, que indica a borda proximal do ligamento transverso do carpo, e estendendo-se até a prega longitudinal radial.

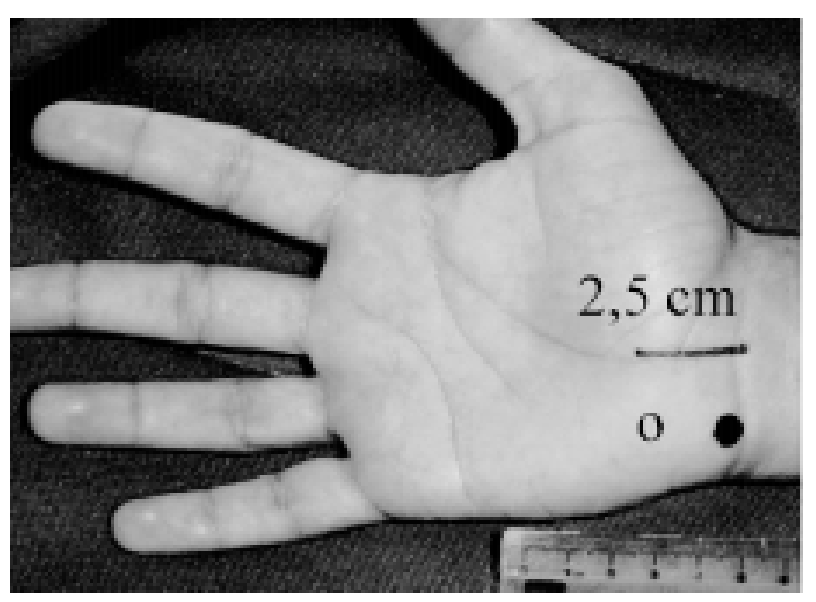

FIGURA 2 - Demarcação da mini-incisão. o) psiforme. 


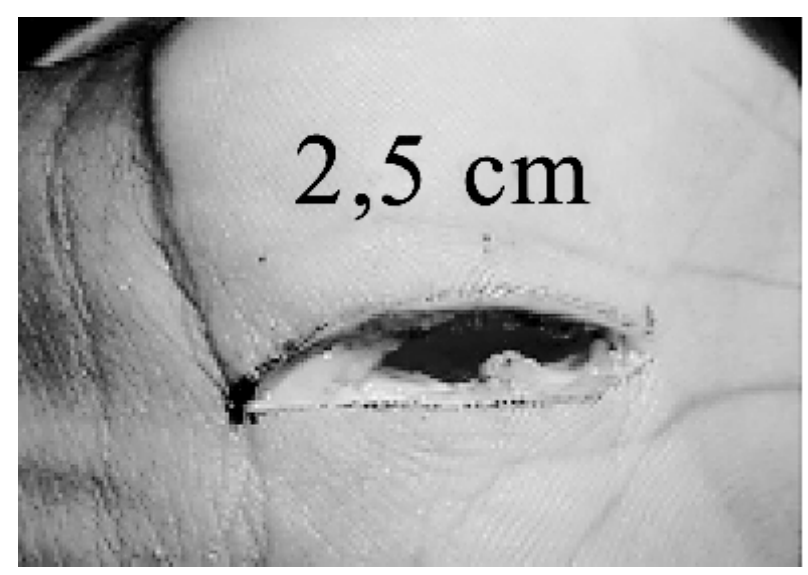

FIGURA 3 - Mini-incisão

Após a incisão na pele, tecido subcutâneo e fáscia palmar, o ligamento carpal transverso era seccionado longitudinalmente em toda sua extensão (Figura 4). Concluído o procedimento a mini-incisão era alongada para avaliar a secção do ligamento carpal transverso, identificação do nervo mediano (Figura 5) e possíveis complicações.

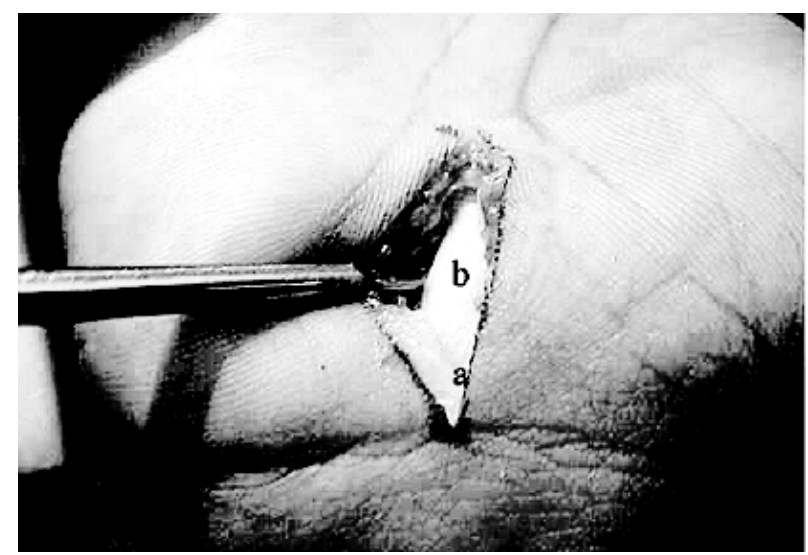

FIGURA 4 - Abertura da mini-incisão.

a) LCT seccionado; b)nervo mediano.

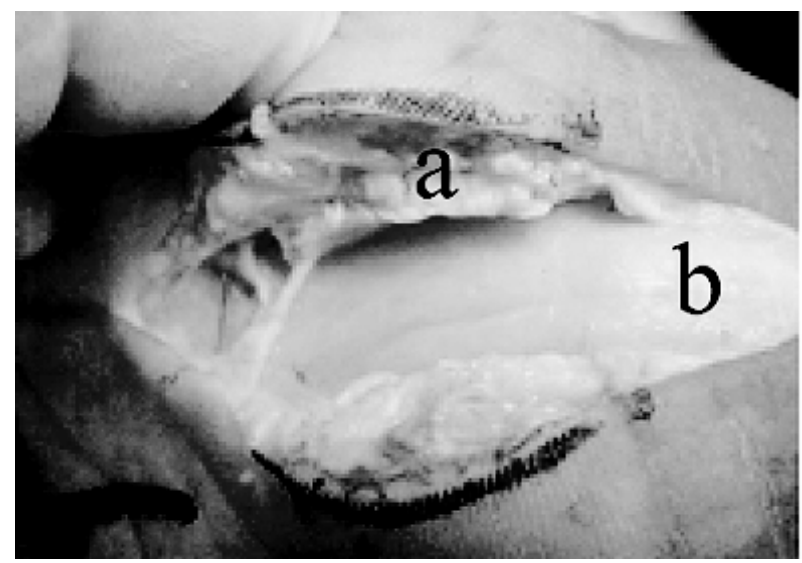

FIGURA 5 - Prolongamento da mini-incisão:

a) LCT; b) nervo mediano.
$\mathrm{Na}$ técnica endoscópica utilizou-se a técnica de dois portais ${ }^{10}$, onde acesso proximal, ou portal de entrada era determinado através de uma linha transversal com $1,0 \mathrm{~cm}$ e distando $1,0 \mathrm{~cm}$ do pisiforme, em direção radial. O acesso distal ou portal de saída era demarcado traçando-se uma linha tangencial no lado ulnar do terceiro quirodáctilo em direção proximal; uma segunda linha era traçada tangencial e no bordo distal do polegar em completa abdução; estas duas linhas formam um ângulo na palma da mão; na bissetriz deste ângulo, a $1,0 \mathrm{~cm}$ proximalmente, marcava-se um ponto, a partir do qual, em sentido radial e transversalmente, estabelecia-se o acesso distal (Figura 6). A incisão na pele era transversal até se identificar a fáscia, onde era realizada uma incisão longitudinal. Com o punho posicionado em extensão máxima, o trocarte com a cânula fenestrada eram introduzidos do acesso proximal para o distal (Figura 7).

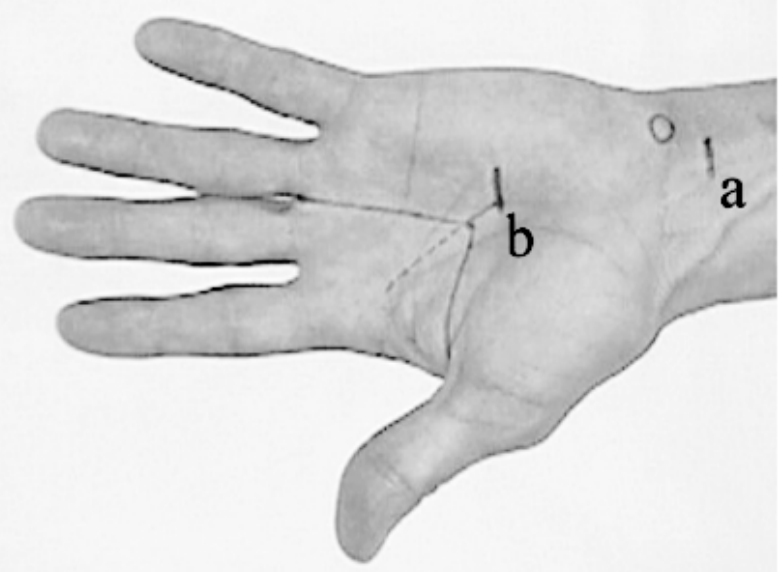

FIGURA 6 - Linhas para demarcação do acesso endoscópico tipo CHOW:

a) portal proximal;o)pisiforme; b)portal distal.

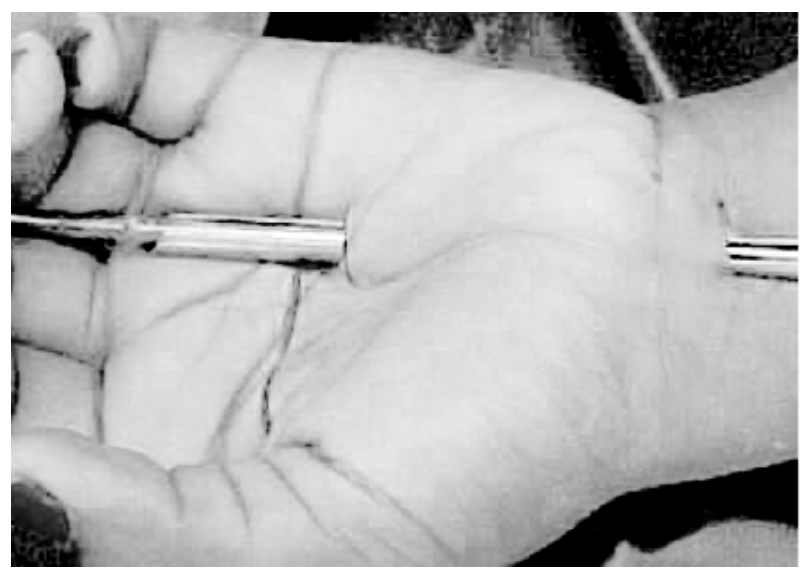

FIGURA 7 - Posicionamento do trocarte e da cânula fenestrada introduzidos entre os dois portais. 
Depois era retirado o trocarte, ficando somente a cânula fenestrada (Figura 8), através da qual era introduzida a óptica do artroscópio (Figura 9) e, com o auxílio do palpador, identificado endoscopicamente o ligamento carpal transverso (Figura 10), o qual era seccionado pelo gancho com lâmina cortante reversa.

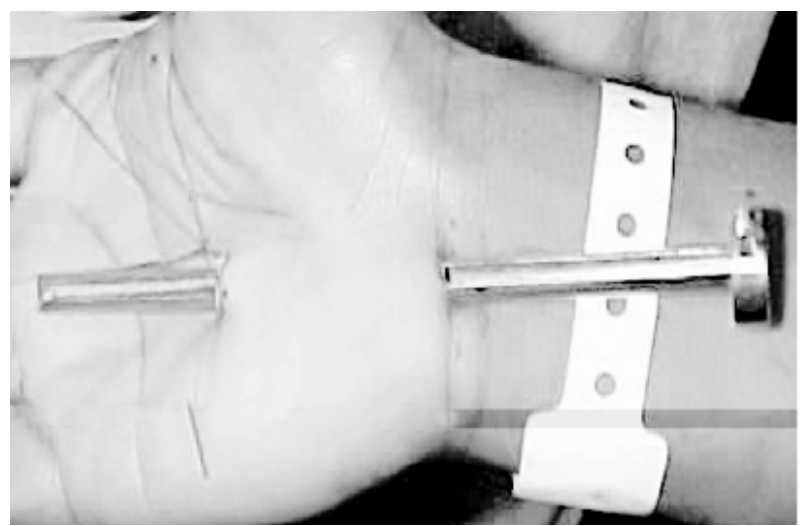

FIGURA 8 - Posicionamento da cânula fenestrada, sem trocarte, introduzida entre os dois portais.

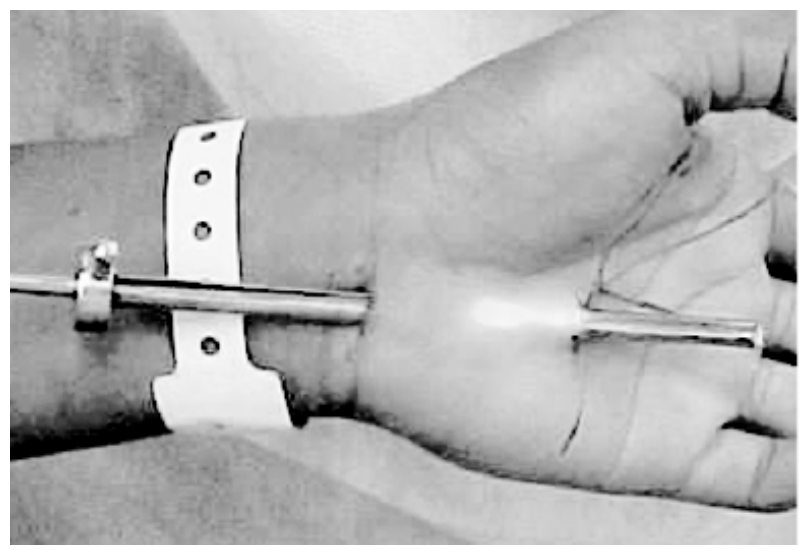

FIGURA 9 - Aspecto da transiluminação cutânea após a introdução da óptica do artroscópio entre os dois portais.

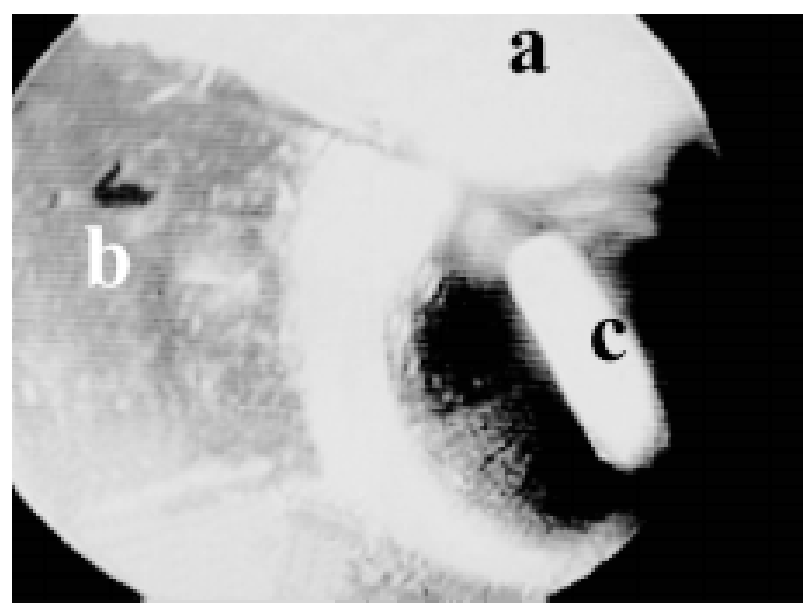

FIGURA 10 - Visão endoscópica: a) LCT; b) cânula fenestrada; c) palpador
Concluído o procedimento endoscópico, efetuavase uma incisão cutânea longa, unindo as duas pequenas incisões transversais, para verificação dos achados; dentre eles, o ligamento carpal transverso ou o nervo mediano íntegro (Figura 11) ou lesado (Figura 12)

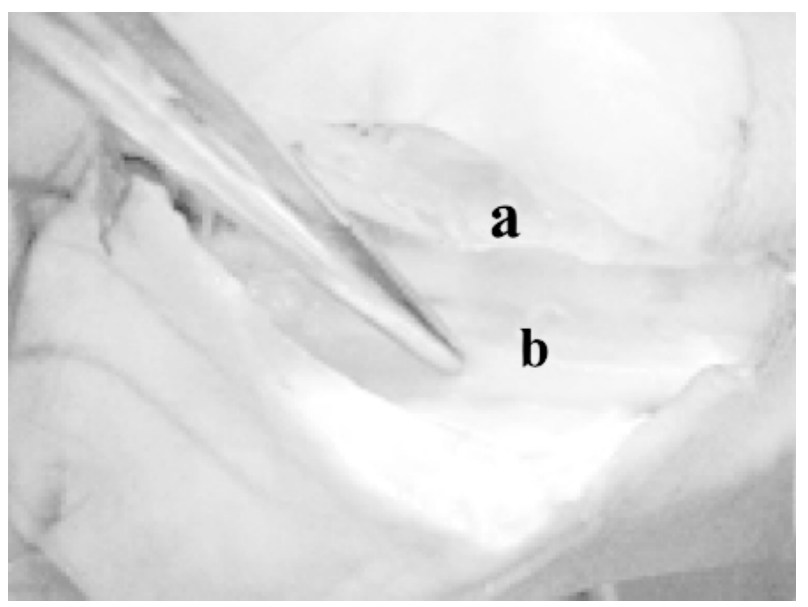

FIGURA 11 - Abertura cutânea entre os dois portais. Observe: a) LCT seccionado; b) nervo mediano.

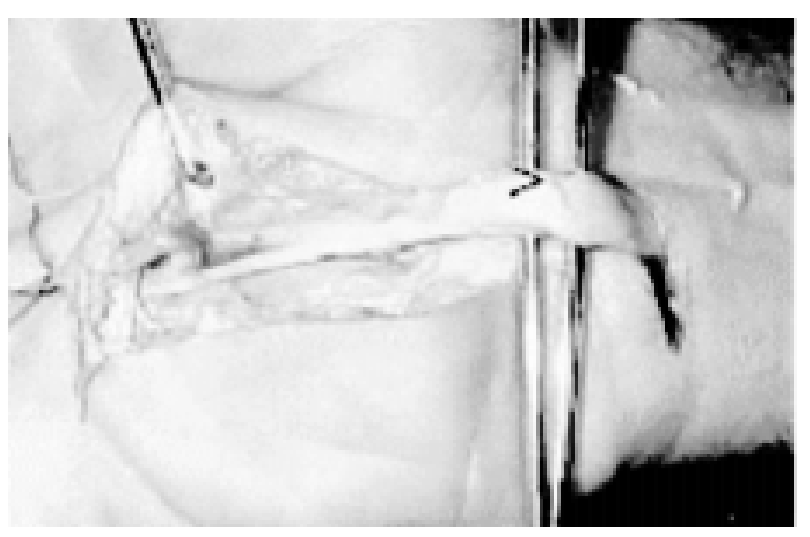

FIGURA 12 - Nervo mediano lesado.

Análise estatística: foram utilizados os seguintes testes na análise dos dados obtidos: teste de Wald para dados categorizados, na análise do resultado da secção do ligamento carpal transverso; teste para diferença de proporções de populações com distribuição normal para análise de complicações após a operação; teste t de Student para diferença de médias para verificar possíveis diferenças estatísticas entre os grupos I e II, em relação à característica tempo operatório. A significância estatística foi fixada em $0,05 \%$ $(\mathrm{p}<0,05)$.

\section{Resultados}

Os resultados encontrados estão delineados nas Tabelas 1 a 6 e nas Figuras 13 a 18 . 
TABELA 1 - Número de casos de secção do ligamento carpal transverso (LCT) por técnica cirúrgica.

\begin{tabular}{ccc}
\hline Seç̧̃o do LCT & Convencional & Endoscópica \\
\hline Completo & 17 & 15 \\
Parcial & 1 & 3 \\
\hline Total & 18 & 18 \\
\hline
\end{tabular}

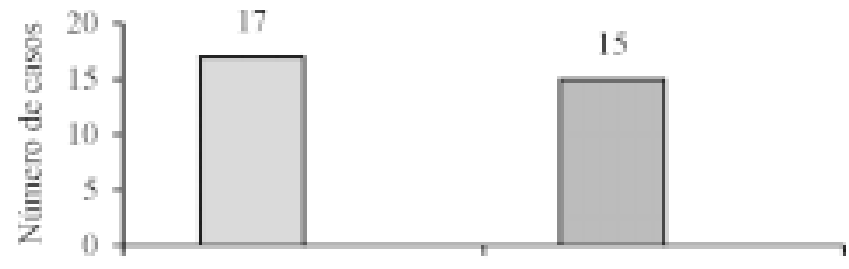

Convencional Endoscópica

Seç̧àio completa

FIGURA 13 - Gráfico que mostra o número de casos de secção do LCT por técnica cirúrgica.

TABELA 2 - Secção LCT por caso e técnica cirúrgica.

\begin{tabular}{l|c|c|c|c|c|c|c|c|c|c|c|c|c|c|c|c|c|c}
\hline Caso & 1 & 2 & 3 & 4 & 5 & 6 & 7 & 8 & 9 & 10 & 11 & 12 & 13 & 14 & 15 & 16 & 17 & 18 \\
\hline LCT: & & & & & & & & & & & & & & & & & & \\
Endoscópica & $\mathrm{P}$ & $\mathrm{P}$ & $\mathrm{P}$ & $\mathrm{C}$ & $\mathrm{C}$ & $\mathrm{C}$ & $\mathrm{P}$ & $\mathrm{C}$ & $\mathrm{C}$ & $\mathrm{C}$ & $\mathrm{C}$ & $\mathrm{C}$ & $\mathrm{C}$ & $\mathrm{C}$ & $\mathrm{C}$ & $\mathrm{C}$ & $\mathrm{C}$ & $\mathrm{C}$ \\
Convencional & $\mathrm{P}$ & $\mathrm{C}$ & $\mathrm{C}$ & $\mathrm{C}$ & $\mathrm{C}$ & $\mathrm{C}$ & $\mathrm{C}$ & $\mathrm{C}$ & $\mathrm{C}$ & $\mathrm{C}$ & $\mathrm{C}$ & $\mathrm{C}$ & $\mathrm{C}$ & $\mathrm{C}$ & $\mathrm{C}$ & $\mathrm{C}$ & $\mathrm{C}$ & $\mathrm{C}$ \\
\hline
\end{tabular}

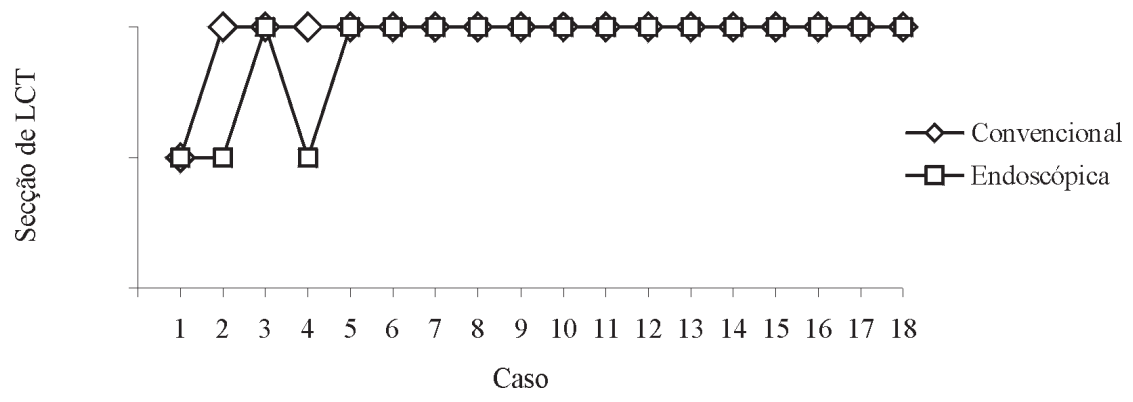

FIGURA 14 - Gráfico que mostra a secção LCT completa (C) ou parcial $(\mathrm{P})$ por caso e técnica cirúrgica.

TABELA 3 - Complicação por caso e técnica cirúrgica.

\begin{tabular}{l|c|c|c|c|c|c|c|c|c|c|c|c|c|c|c|c|c|c}
\hline Caso & 1 & 2 & 3 & 4 & 5 & 6 & 7 & 8 & 9 & 10 & 11 & 12 & 13 & 14 & 15 & 16 & 17 & 18 \\
\hline Endoscópica & $\mathrm{S}$ & $\mathrm{S}$ & $\mathrm{N}$ & $\mathrm{N}$ & $\mathrm{N}$ & $\mathrm{N}$ & $\mathrm{N}$ & $\mathrm{N}$ & $\mathrm{N}$ & $\mathrm{N}$ & $\mathrm{N}$ & $\mathrm{N}$ & $\mathrm{N}$ & $\mathrm{N}$ & $\mathrm{N}$ & $\mathrm{N}$ & $\mathrm{N}$ & $\mathrm{N}$ \\
Convencional & $\mathrm{N}$ & $\mathrm{N}$ & $\mathrm{N}$ & $\mathrm{N}$ & $\mathrm{N}$ & $\mathrm{N}$ & $\mathrm{N}$ & $\mathrm{N}$ & $\mathrm{N}$ & $\mathrm{N}$ & $\mathrm{N}$ & $\mathrm{N}$ & $\mathrm{N}$ & $\mathrm{N}$ & $\mathrm{N}$ & $\mathrm{N}$ & $\mathrm{N}$ & $\mathrm{N}$ \\
\hline
\end{tabular}

$S=\operatorname{Sim} N=$ Não 
Estudo da secção do ligamento carpal transverso comparando as técnicas endoscópica e convencional em cadáver humano

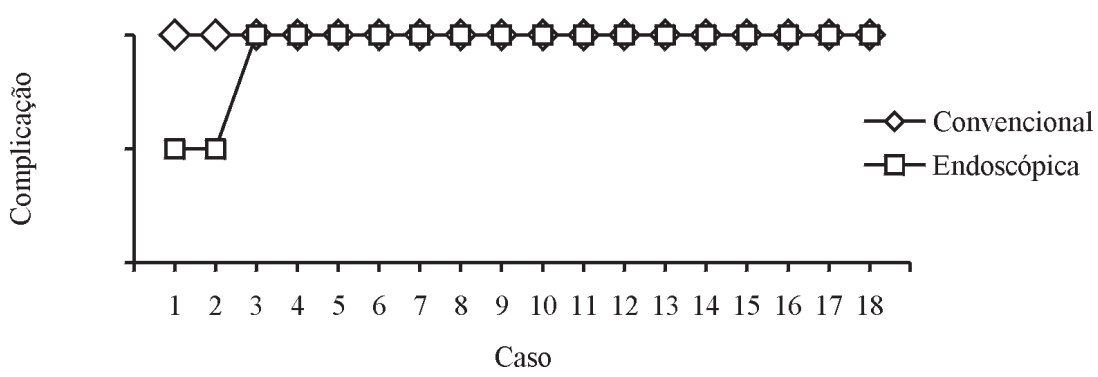

FIGURA 15 - Gráfico que mostra a complicação por caso e técnica cirúrgica.

TABELA 4 - Número de casos com complicações por técnica cirúrgica.

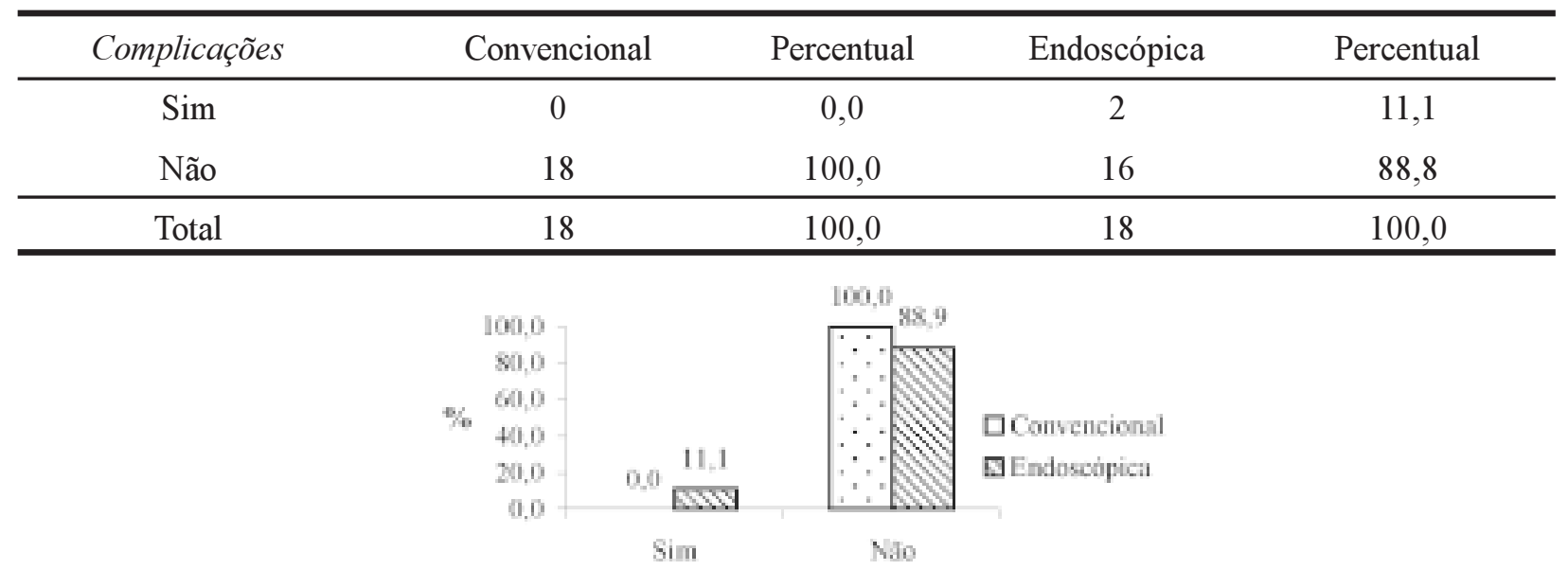

FIGURA 16 - Percentual de casos com complicação por técnica cirúrgica.

TABELA 5 - Estatísticas descritivas do tempo (minutos) de cirurgia por técnica.

\begin{tabular}{lcc}
\hline \multicolumn{1}{c}{ Medidas } & Mini-incisão & Endoscópica \\
\hline Média & 29,72 & 27,22 \\
Erro padrão & 0,64 & 2,03 \\
Desvio padrão & 2,70 & 8,61 \\
Variância da amostra & 7,27 & 74,18 \\
Mínimo & 25,00 & 15,00 \\
Máximo & 35,00 & 45,00 \\
Número de Espécimes & 18 & 18 \\
\hline
\end{tabular}

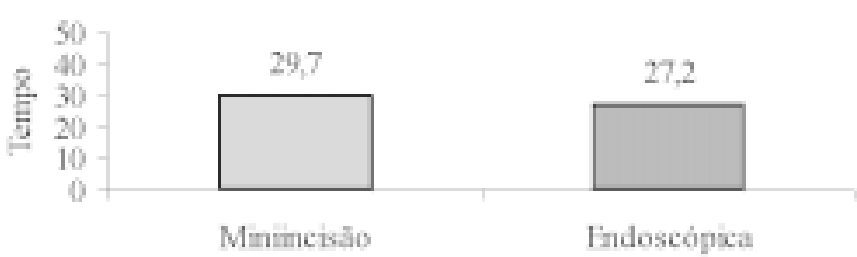

FIGURA 17 - Tempos médios (minuto) de atos operatórios por técnica cirúrgica. 
TABELA 6 - Tempo operatório, em minutos, por caso e técnica cirúrgica.

\begin{tabular}{l|c|c|c|c|c|c|c|c|c|c|c|c|c|c|c|c|c|c}
\hline Caso & 1 & 2 & 3 & 4 & 5 & 6 & 7 & 8 & 9 & 10 & 11 & 12 & 13 & 14 & 15 & 16 & 17 & 18 \\
\hline Endoscópica & 45 & 40 & 30 & 40 & 35 & 30 & 25 & 30 & 25 & 30 & 20 & 20 & 25 & 15 & 20 & 25 & 15 & 20 \\
Mini-incisão & 35 & 35 & 30 & 30 & 30 & 30 & 25 & 30 & 25 & 30 & 30 & 30 & 30 & 30 & 25 & 30 & 30 & 30 \\
\hline
\end{tabular}

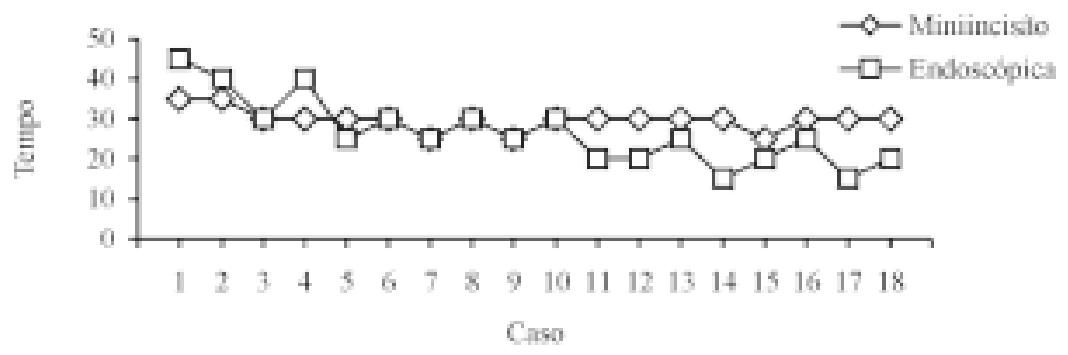

FIGURA 18 - Tempo operatório por caso e técnica cirúrgica.

\section{Discussão}

A síndrome do túnel carpal que consiste na compressão do nervo mediano ao nível do túnel carpal é uma doença marcante porque tem uma alta incidência $(5 \%)$ em trabalhadores que requerem uso repetitivo das mãos. Isto e o fato de ter sido descrita pela primeira vez a cerca de 150 anos, certamente contribui para que ela seja a neuropatia periférica mais diagnosticada e tratada do mundo ${ }^{1}$. Qualquer lesão que reduza de modo significante o tamanho do túnel do carpo pode causar compressão do nervo mediano, produzindo parestesia, anestesia ou hipoestesia nos dedos, com perda progressiva da coordenação e força do polegar e, se a causa da compressão do nervo mediano não for eliminada, resultará em dificuldade de realizar movimentos delicados com o polegar. Como os músculos tenares e os dois músculos lombricais laterais dos outros dedos também são supridos pelo nervo mediano, a movimentação do primeiro ao terceiro dedos pode ser reduzida. Assim, dentre os sintomas mais precoces estão a parestesia noturna e a dor, enquanto a hipotrofia e atrofia da região tênar indica evolução tardia da doença. O diagnóstico é feito através de sintomas clínicos, sinal de Tinel, teste de Phalen e eletromiografia, enquanto o tratamento pode ser conservador ${ }^{2}$ ou cirúrgico, variando de acordo com a gravidade dos sintomas. Quando o tratamento é precoce, indica-se o tratamento conservador que, entre outros, consiste na imobilização por talas de velcro e mudanças nas atividades profissionais. $\mathrm{O}$ tratamento cirúrgico é indicado no insucesso do tratamento conservador ou em graus mais intenso de compressão do nervo mediano e consiste na neurolise deste nervo realizada através da secção completa do ligamento carpal transverso ${ }^{1}$. Para a realização deste procedimento são usadas várias técnicas cirúrgicas que podem ser divididas em dois grupos: os procedimentos cirúrgicos convencionais e os procedimentos endoscópicos, que, por sua vez, apresentam subdivisões ${ }^{3}$. O acesso endoscópico utiliza a visão indireta através de instrumentos de artroscopia modernos ${ }^{8}$ e subdivide-se em dois métodos, conforme emprega um ou dois portais. O método endoscópico que utiliza um portal, ou seja, uma única incisão como entrada e saída, é denominado método de $\mathrm{Agee}^{9}$ enquanto aquele que utiliza dois portais, ou seja, duas incisões, é chamado de método de $\mathrm{Chow}^{10}$. O acesso convencional emprega a visão direta, dispensando instrumentação especial, através de incisões longitudinais no punho, longas ou curtas. A incisão longa dita convencional, mede cerca de $4,5 \mathrm{~cm}^{4}$, podendo chegar ao dobro ${ }^{5}$, enquanto a pequena incisão, também denominada mini-incisão, mede cerca de $2,5 \mathrm{~cm}^{6}$ a $1,5 \mathrm{~cm}^{7}$.

Neste estudo comparativo em cadáver, foi utilizada a técnica endoscópica biportal de Chow, visando segurança e melhor execução do procedimento cirúrgico, enquanto na técnica convencional empregou-se a pequena incisão de $2,5 \mathrm{~cm}$ (mini-incisão), considerando-se melhor visualização do campo operatório e a qualidade da cicatriz.

Korpic e Ladsiedvl demonstraram num estudo comparativo entre os procedimentos cirúrgicos endoscópico e convencionais que a duração da operação era significantemente mais curta quando se utilizou o acesso endoscópico, o que não está de acordo com o presente estudo, onde tal diferença não foi significante ${ }^{13}$. 
Vários autores encontraram secção parcial do ligamento carpal transverso. Uns, utilizando pacientes e estudos comparativos entre os acessos endoscópico e convencional, observaram maior incidência no procedimento endoscópico ${ }^{11}$. Outros, empregando somente o acesso endoscópico, em cadáver, com grande divergência na percentagem de incidência, tais como $55 \%{ }^{14}$ e $0 \%{ }^{12}$. No presente estudo esta incidência foi maior no acesso endoscópico(16,6\%), não significante e ocorreu nos primeiros casos, demonstrando a necessidade de um treinamento em cadáver antes de passar para a fase clínica.

Vários trabalhos mostram lesão do nervo mediano tanto quando utilizaram o acesso endoscópico ${ }^{15}$, como quando empregaram o acesso convencional ${ }^{7,15}$. Neste estudo também foi detectada lesão do nervo mediano, mas somente quando o procedimento endoscópico foi utilizado.

Um trabalho realizado em pacientes utilizando o procedimento aberto tipo mini-incisão não notou melhora significante com a adição da epineurectomia e a tenossinovectomia ${ }^{1}$. Estas duas técnicas adicionais não foram executadas em nenhum dos procedimentos deste trabalho.

Acredita-se, portanto, que os procedimentos cirúrgicos endoscópicos (método de Chow) e convencional (mini-incisão) são eficazes e seguros, desde que o cirurgião tenha um treinamento prévio em cadáver. Seria oportuno um outro estudo comparando as técnicas endoscópica tipo Agee (portal único) e convencional tipo mini-incisão em cadáver humano.

\section{Conclusão}

Não há diferença significante no que se refere à secção do ligamento carpal transverso e tempo operatório quando se comparam as técnicas endoscópica tipo Chow e convencional tipo mini-incisão. A incidência de lesão do nervo mediano, porém, é significante $(\mathrm{p}<$ $0,05)$ quando se utiliza o acesso endoscópico, o que pode estar relacionado à curva de aprendizagem.

\section{Referências}

1. Severo A, Ayzemberg H, Pitagora T, Nicolodi D, Mentz L, Osvandre L. Síndrome do túnel carpal: análise de 146 casos operados pela miniincisão. Rev Bras Ortop 2001; 36(9): $330-5$.

2. Pereira ES, Zallman I, Mori CE, Amard JT. Síndrome do túnel do carpo: análise comparativa entre tratamento conservador e cirúrgico. Rev Bras Ortop 1993; 28:570-8.

3. Schmidt W, Gruber AA, Hammer R. Results of different incisions in treatment of carpal tunnel syndrom. Handchir Mikrochir Plast Chir 2000; 32(1): 67-9.

4. Richter VM, Bruser P. Surgical treatment of carpal tunnel syndrome: a comparison between long and short incision and endoscopic release. Handchir Mikrochir Plast Chir1996; 28(3): $160-6$

5. Wilson KM. Double incision open technique for carpal tunnel release: an alternative to endoscopic release. J Hand Surg 1994; 19(6): 907-12

6. Ortiz J, Lobet AJ. Síndrome do canal carpiano: tratamento cirúrgico por miniincisão. Rev Bras Ortop 1990; 25: 50-4.

7. Lee WP, Strickland JW. Safe carpal tunnel release via a limited palmar incision. Plast Reconstr Surg 1998; 10(2): 418-24.

8. Sampaio ACD. Bases anatômicas e cirúrgicas da liberação endoscópica do túnel cubital: sistematização das estruturas internas [Tese - Mestrado]. Universidade Federal do Ceará; 1997.

9. Agee JM, Mccarroll HRJr, Tortosa RD, Berry DA, Szabo RM, Peimer C. Endoscopic release of the carpal tunnel: a randomized prospective multicenter study. J Hand Surg 1992; 17A 987-95

10. Chow JCY. Endoscopic releaseof the carpal tunnel ligament:a new technique for carpal tunnel syndrom. J Artroscopy 1989; 5: 19 .

11. Hulsizer D. L.; Staebler, M. P.; Weiss, A. P.; Akelman, E. The results of revision carpal tunnel release following previous open versus endoscopic surgery. J Hand Surg 1998; 23(5): $865-9$

11. Jacobsen MB, Rahme H. A prospective randomized study with au independent observer comparing open carpal tunnel release with endoscopic carpal tunnel release. J Hand Surg 1996; 21(2): 202-4

13. Korpik G, Ladsiedvl F. Carpal tunnel syndrom: a comparison of endoscopic and open surgical treatment. Handchir Mikrochir Plast Chir 1996; 28(3): 133-7.

14. Heest AV, Waters P, Simmons B, Schwartz JT. A cadaveric study of the single-portal endoscopic carpal tunnel release. J Hand Surg 1995; 20A(3): 363-6.

15. Palmer AK, Toivonen DA. Complication of endoscopic and open carpal tunnel release. J Hand Surg 1999; 24(3): 561-5. 
$\overline{\text { Estrela Neto J, Leite JAD, Bezerra MJC. Evaluation of surgical section of transverse carpal ligament }}$ comparing endoscopic and conventional techniques in human corpses. Acta Cir Bras [serial online] 2003 Mar-Apr;18(2). Available from URL: http://www.scielo.br/acb.

ABSTRACT - Purpose: The aim of this study is to evaluate, in human corpses, the section of the transverse carpal ligament by comparing endoscopic (Chow's technique) and conventional (miniincision) accesses. Methods: Eighteen fresh not claimed corpses were distributed into 2 groups: group 1 (18 left wrists) and group 2 (18 right wrists). Group 1 was subjected to endoscopic access (Chow's technique) while in Group 2 ligaments were accessed through mini-incisions. Results: Complete section of transverse carpal ligament was attained in 15 cases $(83,3 \%)$ in group 1 and 17 cases $(94,4 \%)$ in group 2. Operative time in group 1 averaged 27,2 minutes and 29,2 minutes in group 2. Lesion of median nerve occurred in 2 cases (11,1\%) in group 1. Group 2 presented no nerve lesions. Conclusion: It is concluded that there is no significant difference when comparing endoscopic and conventional techniques in the section of transverse carpal ligament. The median nerve injury, inspite of its significant $(\mathrm{p}<0,05)$ incidence in this study may be related to the researcher's learning curve.

KEY WORDS - Carpal tunnel syndrome. Endoscopic versus conventional technique.

Conflito de interesse: nenhum

Fonte de financiamento: nenhuma

Correspondência:

José Estrela Neto

Rua Dr. José Lourenço, 1.500/102-B

60115-281 Fortaleza - Ceará.

Tel: (85)261-0435 / 9983-4544

joseestrela@globo.com

Data do recebimento: 04/01/2003

Data da revisão: 21/01/2003

Data da aprovação: 08/02/2003 